\title{
Evolution and genome specialization of Brucella suis biovar 2 Iberian lineages
}

\author{
Ana Cristina Ferreira ${ }^{1,2^{*}}$, Rogério Tenreiro $^{2}$, Maria Inácia Corrêa de Sá ${ }^{1}$ and Ricardo Dias ${ }^{2}$
}

\begin{abstract}
Background: Swine brucellosis caused by B. suis biovar 2 is an emergent disease in domestic pigs in Europe. The emergence of this pathogen has been linked to the increase of extensive pig farms and the high density of infected wild boars (Sus scrofa). In Portugal and Spain, the majority of strains share specific molecular characteristics, which allowed establishing an Iberian clonal lineage. However, several strains isolated from wild boars in the NorthEast region of Spain are similar to strains isolated in different Central European countries.

Results: Comparative analysis of five newly fully sequenced B. suis biovar 2 strains belonging to the main circulating clones in Iberian Peninsula, with publicly available Brucella spp. genomes, revealed that strains from Iberian clonal lineage share $74 \%$ similarity with those reference genomes. Besides the $210 \mathrm{~kb}$ translocation event present in all biovar 2 strains, an inversion with $944 \mathrm{~kb}$ was presented in chromosome I of strains from the lberian clone. At left and right crossover points, the inversion disrupted a TRAP dicarboxylate transporter, DctM subunit, and an integral membrane protein TerC. The gene dctM is well conserved in Brucella spp. except in strains from the Iberian clonal lineage. Intraspecies comparative analysis also exposed a number of biovar-, haplotype- and strain-specific insertion-deletion (INDELs) events and single nucleotide polymorphisms (SNPs) that could explain differences in virulence and host specificities. Most discriminative mutations were associated to membrane related molecules (29\%) and enzymes involved in catabolism processes (20\%). Molecular identification of both B. suis biovar 2 clonal lineages could be easily achieved using the target-PCR procedures established in this work for the evaluated INDELS.
\end{abstract}

Conclusion: Whole-genome analyses supports that the B. suis biovar 2 lberian clonal lineage evolved from the CentralEuropean lineage and suggests that the genomic specialization of this pathogen in the Iberian Peninsula is independent of a specific genomic event(s), but instead driven by allopatric speciation, resulting in the establishment of a new ecovar.

Keywords: Brucella spp., B. suis biovar 2, Phylogenomics, comparative genomics, Iberian ecovar

\section{Background}

Brucella suis is a facultative intracellular pathogen infecting a broad range of animals and humans. This species comprises five biovars, denominated biovar 1 to 5 , which infect specific hosts. Different molecular approaches have shown a considerable diversification within the $B$. suis clade and probable relationships among the biovars and their preferential hosts were suggested [1-4]. Biovars 1, 2 and 3 infect suidae and are the etiological agents of swine brucellosis, but biovars 1 and

\footnotetext{
*Correspondence: cristina.ferreira@iniav.pt
'Instituto Nacional de Investigação Agrária e Veterinária, I.P. (INIAV, IP), Av. da

* Correspondence: cristina.ferreira@iniav.pt
'Instituto Nacional de Investigação Agrária e Veterinária, I.P. (INIAV, IP), Av. da República, Quinta do Marquês, 2780-157 Oeiras, Portugal

Universidade de Lisboa, Faculdade de Ciências, Biosystems and Integrative Sciences Institute (BioISI), Edificio TecLabs, Campus da FCUL, Campo Grande, 1749-016 Lisbon, Portugal
}

(c) The Author(s). 2017 Open Access This article is distributed under the terms of the Creative Commons Attribution 4.0 International License (http://creativecommons.org/licenses/by/4.0/), which permits unrestricted use, distribution, and reproduction in any medium, provided you give appropriate credit to the original author(s) and the source, provide a link to the Creative Commons license, and indicate if changes were made. The Creative Commons Public Domain Dedication waiver (http://creativecommons.org/publicdomain/zero/1.0/) applies to the data made available in this article, unless otherwise stated. from biovar 2. In the public health context, biovar 2 is rarely pathogenic, whereas biovars 1 and 3 are highly pathogenic and cause severe disease in humans [5]. $B$. suis biovar 2 was only described in Europe and also infects hares. It is generally accepted, that wild boars (Sus scrofa) and European hares (Lepus europaeus) are both wildlife reservoirs of this biovar and the source of transmission to backyard and extensively reared pigs [6]. Previous studies on the genetic structure of the $B$. suis biovar 2 population by Multiple Locus Variable-number Tandem Repeat Analysis (MLVA) [4, 7-10], PCR-RFLP analysis of omp31, omp $2 a$ and omp $2 b$ genes [10-13] and whole-genome optical mapping [11] supports the existence of two circulating lineages in the Iberian Peninsula (Portugal and Spain): a Central-European and a Iberian 
clonal lineage. The latter has been described exclusively in the Iberian Peninsula, in pigs and wild boars, mainly south of the River Ebro, suggesting a genomic specialization and local adaptation [12].

Pathogen fitness may vary from host to host. The way that pathogens evolve, within or outside hosts, and the strategy used by the host to resist infection can be determinant for pathogen specialization and an important driver of virulence evolution. Host specificity is associated with a number of genomic signatures, including genomic degeneration and genomic rearrangements [14]. Recently, the complete genome sequences of five $B$. suis biovar 2 strains representative of Iberian and Central-European clonal lineages, isolated from wild boars in Portugal and Spain, have been released $[15,16]$, and it was shown that the Iberian lineage is characterized by the presence of a large chromosomal inversion [11]. Whole genome sequencing (WGS) has opened new insights into brucellae taxonomy and phylogeny. The genomic era allowed developing a plethora of new high-resolution methods for the detection and typing of pathogens, and opened new avenues for understanding the full ecological diversity of the genus Brucella. Over the last decade, six new species were included in the genus [17]. Further, "atypical" Brucella strains were isolated from diverse animal sources, including cold-blooded hosts, such as frogs and fish [18-20] and are likely going to be proposed as new species in the future.

In this study, we performed a comparative analysis of the genomes of B. suis biovar 2 with other Brucella species to disclose genomic and structural differences between Iberian and Central-European clones. This will allow the assessment of potential factors favoring evolution towards host specialization and contribute to a better understanding of the mechanisms underlying evolution and specialization of Iberian lineages.

\section{Methods}

\section{Bacterial strains and genetic characterization}

A total of 190 Brucella spp. strains were used for PCR validation assays, including 22 reference strains representative of eight Brucella species and 168 B. suis isolates. Eleven of these isolates belonged to biovar 1, 152 to biovar 2, one to biovar 3 and four to biovar 4. Among the B. suis biovar 2 isolates, 104 were representative of the Iberian clonal lineage and 48 of the CentralEuropean clonal lineage. Phenotypic characterization of all Brucella spp. isolates was performed as previously described [21]. All B. suis isolates were previously subjected to PCR-RFLP analysis of the omp $2 a$ and omp $2 b$ [22] and omp31 [23] genes, to assess the different haplotypes, and to target-PCR to confirm the presence of the large inversion in $B$. suis biovar 2 Iberian clonal lineage [11]. All data regarding strain characterization are presented in Additional file 1: Table S1.

\section{Comparative genomic analysis}

The analysis involved 25 brucellae complete genomes (Additional file 2: Table S2), including the five recently sequenced $B$. suis biovar 2 isolates (strains PT09143, PT09172, Bs143CITA, Bs364CITA and Bs396CITA - for details on sequencing, assembly and annotation see Additional file 3 and Additional file 4, Tables S3 and S4).

Multiple sequence alignment of concatenated chromosomes I and II was performed by a superstretch approach using as reference the annotated sequence of strain B. suis ATCC 23445: DNA seed 10 matches in windows size of 25 bases, minimal stretch length 60 bases, minimal cut-off for stretch identity of $60 \%$ in screening windows of 30 bases was used. The mutation search was performed into the multiple sequence alignment, and included single nucleotide polymorphisms (SNPs; including silent, missense and intergenic mutations) and insertions/deletions (INDELs) variants. To avoid false positives on mutation calling, optimized alignment overlapping stretches settings were used. All query sequences were screened against the reference sequence. A window size of $10 \mathrm{bp}$ was used for determination of pairwise distance, clustering and quality scores for each mutation. The cut-off value for mutation calling on each alignment was set to the 90th percentile of the Poison distribution for score values obtained to each type of mutation. Multiple sequence alignment and mutation analysis were conducted in Kodon V3.62 (Applied Maths, Belgium).

Functional enrichment analysis for missense SNPs among $B$. suis clonal linages was performed on the consolidated semantic annotation obtained from Gene Ontology (GO) [24], the integrative protein signature database (InterPro) [25], and Kyoto Encyclopedia of Genes and Genomes (KEGG) [26] databases, using Blast2GO pipeline (version 2.7.1) [27].

\section{Evolutionary whole-genome-based studies}

To understand the impact of genomic structural rearrangements in the brucellae phylogeny, the relationships among the 25 Brucella spp. genomes (Additional file 2: Table S2) were determined using WG-MSA. Clustering analysis was performed using Unweighted Pair Group Method using Arithmetic averages (UPGMA). All positions containing gaps and missing data were eliminated. Chromosomal alignment and clustering analysis were conducted in Kodon V3.62.

The evolutionary history was inferred from genomewide SNPs (WG-SNP) using Neighbour-Joining (NJ) and Maximum Likelihood (ML). The analysis included the 23 genomes of the eight accepted species and the genome of Brucella sp. strain 09RB8910 was used as outgroup (Additional file 2: Table S2). The initial trees were drawn to scale, with branch lengths in the same units as those of the evolutionary distances used to infer the phylogenetic trees. All positions containing gaps and 
missing data were eliminated. Final phylogenetic trees were built using ML method based on the Tamura-Nei model. The evolutionary analyses were conducted in MEGA6 software package [28].

Evolutionary relationships were also inferred from INDELs information obtained from WG-MSA and coded as binary characters ( 1 if gap present, 0 if absent). Each instance in the $0 / 1$ matrix corresponds to a single INDEL character, which may reflect either an insertion or a deletion relatively to reference sequence. The quality score was optimized based on a set of parameters to discriminate effective INDELS: quality score, INDEL size, number of neighboring SNPs and INDELs, position of the second different base pair, and INDELs distance to the edge on assembled contig. Likewise, only INDELs with quality scores $<1$ and sizes bigger than $15 \mathrm{bp}$ were considered. The complete set of the 25 brucellae genomes were included in the analysis (Additional file 2: Table S2). A minimum spanning tree (MST) was generated from a subset of random INDELs using BioNumerics version 6.6 (Applied Maths, Belgium).

\section{PCR assessment of INDEL events}

All the INDELs found to be discriminative of B. suis biovar 2 Iberian clonal lineage were assessed by PCR in the collection of the previously described 190 Brucella strains. Primers for each INDEL were designed using the nucleotide positions relative to the reference strain ATCC 23445 and specificity of all the primers was confirmed by BLAST analysis against the published genome sequence of the reference strain. The primer sequences and expected amplicon sizes are provided in Table 1. Genomic DNA from each strain was prepared using the High Pure PCR Template Preparation Kit (Roche Diagnostics, Mannheim, Germany) according to the manufacturer. PCR amplification was performed in a total volume of $25 \mu \mathrm{l}$ containing $25 \mathrm{ng}$ of DNA, 10× PCR Reaction Buffer, $1 \mathrm{U}$ of Taq DNA polymerase (Promega, USA), $200 \mathrm{mM}$ of each dNTPs and $0.3 \mathrm{mM}$ of each flanking primers. Amplifications were performed in a MyCycler thermal Cycler (Bio-Rad, France). An initial denaturation step at $95{ }^{\circ} \mathrm{C}$ for $3 \mathrm{~min}$ was followed by 30 cycles of denaturation at $95{ }^{\circ} \mathrm{C}$ for $20 \mathrm{~s}$, primer annealing at $56{ }^{\circ} \mathrm{C}$ for $30 \mathrm{~s}$ and elongation at $72{ }^{\circ} \mathrm{C}$ for $30 \mathrm{~s}$. The final extension step was performed at $72{ }^{\circ} \mathrm{C}$ for $5 \mathrm{~min}$. Five microliter of amplification products were loaded on a $2 \%$ standard agarose gel and run under a voltage of $8 \mathrm{~V} / \mathrm{cm}$ for $60-90 \mathrm{~min}$. A 100-bp ladder (Invitrogen, USA) was used as molecular size marker.

\section{Results and discussion}

\section{Phylogenomic relationships of $B$. suis biovar 2}

To understand the genomic specialization observed in the Iberian Peninsula, the genetic structure and evolutionary relationships of the $B$. suis strains isolated in the Iberian Peninsula were assessed by three Whole-Genome based phylogenetic approaches: Multiple Sequence Alignment (WG-MSA); Single Nucleotide Polymorphism distribution (WG-SNP) and insertion and deletion events distribution (WG-INDEL). The phylogenetic analyses involved the full sequence of the five recently published genomes of $B$. suis biovar 2 isolates, and 20 brucellae complete genomes available at the time of the analysis. All genomes with the exception of B. suis 686 (biovar 3) have two circular chromosomes (Additional file 2: Table S2).

The WG-MSA analysis was performed for the concatenated chromosomes of each genome and the resultant similarity matrix was used for clustering of the 25 genomes in order to assess the effect of the genomic structural organization on brucellae phylogeny. Brucella spp. genomes were grouped into two highly distinct clusters (Additional file 5: Figure S1). It is generally

Table 1 List of the primers used for assessment of the INDEL events differentiating the two B. suis biovar 2 clonal lineages

\begin{tabular}{|c|c|c|c|c|c|c|c|}
\hline \multirow[t]{2}{*}{ INDEL } & \multicolumn{2}{|c|}{ Primer sequence $5^{\prime} \rightarrow 3^{\prime}$} & \multirow[t]{2}{*}{ Chr } & \multirow[t]{2}{*}{$\begin{array}{l}\text { Amplified region } \\
\text { in ATCC } 23445\end{array}$} & \multirow[t]{2}{*}{$\begin{array}{l}\text { Affected CDS in } \\
\text { ATCC } 23445\end{array}$} & \multicolumn{2}{|c|}{$\begin{array}{l}\text { PCR product expectec } \\
\text { size }(b p)^{a}\end{array}$} \\
\hline & & & & & & $\overline{\mathrm{CECL}}$ & $\mathrm{ICL}$ \\
\hline SI79506 & $F R$ & $\begin{array}{l}\text { tgcacacgtagggtcgata } \\
\text { cacccagatgttcggctat }\end{array}$ & । & $79,420 . .80357$ & BSUIS_A0075 & 938 & 260 \\
\hline SI1356057 & $\mathrm{FR}$ & taggctgcctggaattcatc cggcaagttcacctctgact & । & $1,355,436 . .1356288$ & Intergenic & 853 & 765 \\
\hline SI1423448 & $\mathrm{FR}$ & tttcttattccacccgatcc caaaatgttactgcgtgaagc & । & $1,423,358 . .1423655$ & Intergenic & 298 & $298(218)$ \\
\hline SI1627485 & $\mathrm{FR}$ & $\begin{array}{l}\text { caatccacaggagatcggt } \\
\text { gcttcacgggtatcatgtaca }\end{array}$ & 1 & $1,627,421 . .1,627,600$ & BSUIS_A1714 & 180 & 129 \\
\hline SI2041144 & $\mathrm{FR}$ & tcggaaatggacgaatatca ttcttgtcgtcggaaatgtc & $\|$ & $107,306.107533$ & Intergenic & 228 & $228(171)$ \\
\hline SI2603410 & $F R$ & agtggattttggtgcgtttc aagatgagcgggaaatgttg & $\|$ & $679,423.680774$ & BSUIS_B0700; BSUIS_B0701 & 1352 & 508 \\
\hline LI3234619 & $\mathrm{FR}$ & tattattcactttgagcggca aactgcaaaagcttggctg & $\|$ & $1,310,801 . .1312970$ & BSUIS_B1354-BSUIS_B1357 & 2170 & 174 \\
\hline
\end{tabular}

CECL Central-European clonal lineage

Chr chromosome

ICL Iberian clonal lineage

${ }^{a}$ Values in parenthesis only refer to haplotype $2 e$ strains 
accepted that chromosomal inversions or translocations events may affect the identity values even though little impact is observed in gene content [29]. Indeed, B. abortus presents an inversion of $640 \mathrm{~kb}$ in chr II [2, 30, 31] and B. suis biovar 2 strains PT09172, PT09143 and Bs143CITA present an inversion in chr I with approximately $944 \mathrm{~kb}$ [11], as well as the known $210 \mathrm{~kb}$ segment of chr I translocated to chr II $[1,2,11]$. Moreover, strain 686 from B. suis biovar 3, the unique reference strain with a single chromosome of $3.3 \mathrm{Mbp}$ [32], also revealed an inversion of $790 \mathrm{~kb}$, located between nucleotide \#285,192 and \#1,074,164 (ATCC 23445 reference positions), in a similar region as the inversion occurring in biovar 2 strains from the Iberian clonal lineage. To the best of our knowledge, this is the first description of a genomic inversion in B. suis biovar 3 and of chromosomal structural rearrangements as evolutive features shared by $B$. suis biovar 2 and biovar 3 strains.

To unveil the evolutionary history of B. suis biovar 2 imprinted on the genomic background, WG-SNP analysis was performed to remove the effect of chromosomal rearrangements (translocations and inversions) on $B$. suis phylogeny. The evolutionary history was inferred from a total of 114,031 putative SNPs shared among the 23 more closely related genomes and the genome of the amphibian isolate 09RB8910 (Fig. 1-a; Additional file 6: Table S5). To get a more detailed view on $B$. suis phylogeny, a second tree was built only considering the 30,255 putative SNPs shared among the more closely related Brucella species (Fig. 1-b). The evolutionary distances were computed assuming equality of substitution pattern among lineages and of substitution rates among sites using the Maximum Composite Likelihood method and clustered by NeighborJoining method (Fig. 1). The unrooted tree sorts the brucellae genomes into eight clades, as follows: the $B$. suis biovar 2 clade (A); the B. suis-B. canis clade (B); the B. suis biovar 5 clade $(C)$; the $B$. microti clade (D); the B. ovis clade (E); the B. pinnipedialis-B. ceti clade (F); the $B$. abortus clade (G), and the B. melitensis clade (H) (Fig. 1-b). These results are in accordance with several phylogenetics studies using WG-SNP or MLST data [2, 3, 33-35]. The B. suis biovar 2 isolates formed two distinct phylogenetic

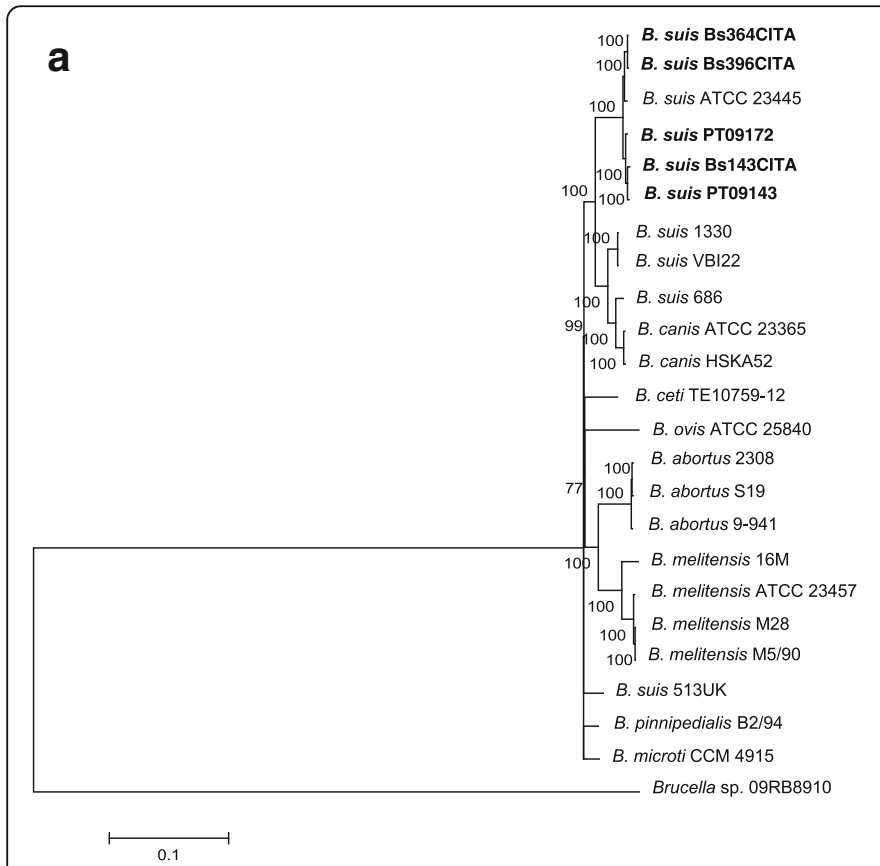

b

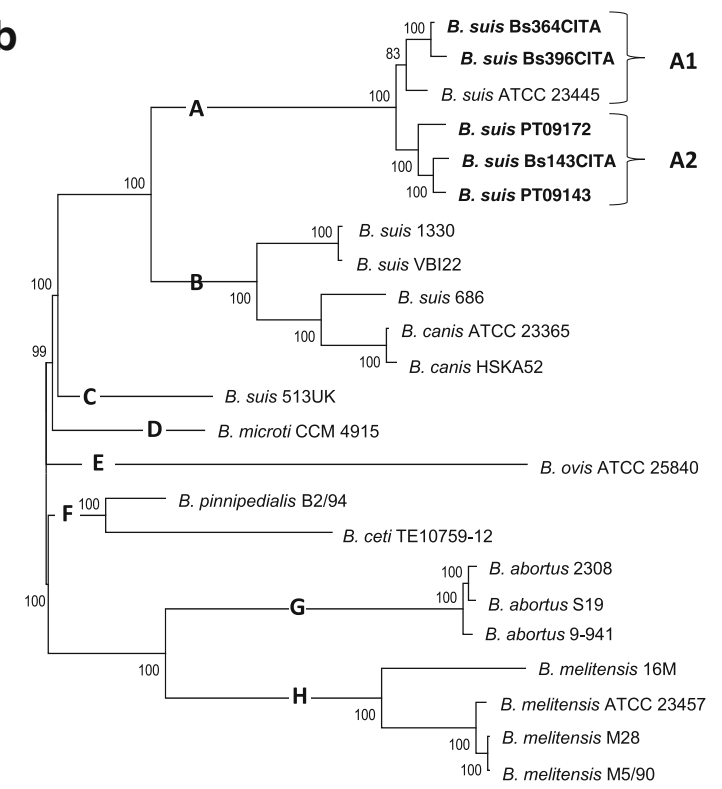

Fig. 1 Evolutionary relationships inside genus Brucella inferred from WG-SNP analysis. a Rooted phylogenetic tree with Brucella sp. 09 RB8910 as outgroup. The evolutionary history was inferred by using the Maximum Likelihood method based on the Tamura-Nei model [1]. The tree with the highest log likelihood $(-581,757.1726)$ is shown. The percentage of trees in which the associated taxa clustered together is shown next to the branches. Initial tree(s) for the heuristic search were obtained automatically by applying Neighbor-Join and BioNJ algorithms to a matrix of pairwise distances estimated using the Maximum Composite Likelihood (MCL) approach, and then selecting the topology with superior log likelihood value. The tree is drawn to scale, with branch lengths measured in the number of substitutions per site. The analysis involved 25 nucleotide sequences. There were a total of 114,041 positions in the final dataset. $\mathbf{b}$ Unrooted phylogenetic tree showing in more detail the 23 core Brucella genomes, involving a total of 30,255 SNPs in the final dataset and depicting eight clades (A to H). The evolutionary history was inferred by using the Maximum Likelihood method. The tree with the highest log likelihood $(-193,253.4180)$ is shown. The percentage of trees in which the associated taxa clustered together is shown next to the branches. Initial tree(s) for the heuristic search were obtained automatically by applying Neighbor-Join and BioNJ algorithms to a matrix of pairwise distances estimated using the Maximum Composite Likelihood (MCL) approach, and then selecting the topology with superior log likelihood value. The tree is drawn to scale, with branch lengths measured in the number of substitutions per site. Evolutionary analyses were conducted in MEGA6 
groups in clade A, one corresponding to the CentralEuropean clonal lineage (subclade A1) and the other to the Iberian clonal lineage (subclade A2), thus giving additional phylogenetic support at genome level for the occurrence of these clonal lineages already revealed by chromosome I optical maps [11]. Moreover, the evolutionary history inferred from the $944 \mathrm{~kb}$ inversion region distinctive of subclade A2 and from $210 \mathrm{~kb}$ translocated region of clade A showed no differences in comparison to whole-genome analysis. Alike topologies were observed for coding and non-coding regions (data not shown). Thus, the mechanisms for genomic specialization on the Iberian lineages seem to be independent of the chromosomal rearrangement events observed in these clades.

The WG-SNP approach relies on reference-based mapping and does not detect variations in regions that are not present in the reference sequence (i.e. ATCC 23445), and is therefore only suitable for comparison of the core genome shared among all strains. WG-INDELs are largely ignored in phylogenetic reconstruction but provide a suite of markers complementary to nucleotide substitutions with enormous potential for molecular phylogenetics [36-38]. A total of 1, 131 INDELs were revealed in the analysis (Additional file 6: Table S6). In order to confirm the phylogenetic relationships suggested by the former approaches, a minimum spanning tree (MST) was generated from a data set of 255 representative INDELs, which included seven Iberian specific INDELs and a subset of 248 randomly chosen from the 1131 INDELs. Evolutionary relationships based on WGINDELs are congruent with those from WG-SNP analysis, further revealing B. suis biovar 2 Central-European clonal lineage genomes allocated in a well-defined cluster, from which the other B.suis biovars and Brucella species seem to evolve (Fig. 2). Therefore, we can speculate that biovar 2 Iberian clonal lineage evolved from the Central-European clonal lineage, representing an ongoing allopatric speciation process as described for other

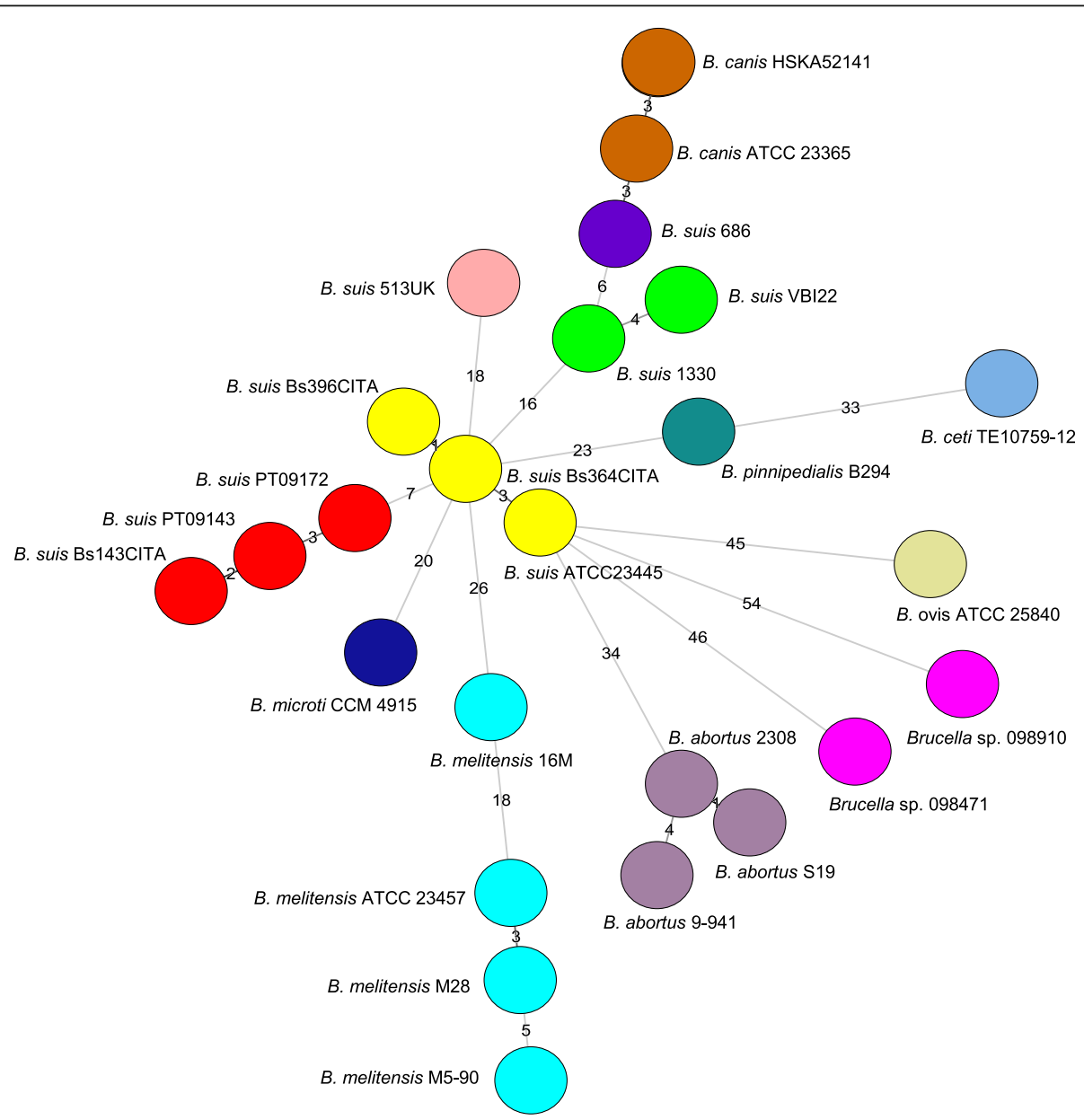

Fig. 2 Minimum spanning tree depicting the genomic relationships of Brucella species and based on WG-INDELs analysis. A data set of 255 representative INDELs was used, including 7 Iberian ecovar specific indels and a subset of 248 randomly chosen INDELs from the 1131 indels obtained from the comparative analysis of the 25 brucellae genomes. Colour codes are associated with Brucella spp. groups and the number of changes between patterns is presented. The MST was constructed with a categorical coefficient using BioNumerics version 6.6 
specialized pathogenic bacteria [38-40]. Since the Iberian clonal lineage is only found in Portugal and Spain south of the Ebro river and the Central-European clonal lineage is only found above this geographical barrier (northeast Spain and other European countries), an ecological differentiation seems to have occurred resulting in the establishment of a new ecovar.

\section{Comparative genomics of $B$. suis biovar 2}

High homology (>98\%) between Brucella spp. are normally found in association with their preferred hosts and has apparently resulted in adaptive changes over time [41]. Phylogenomic relationships have supported the existence of two B. suis biovar 2 phylogenetic groups, which are well separated from $B$. suis biovar 1 and 3 . Therefore, the comparative genomics analysis was focused on the detection of distinctive genetic events between those two lineages in comparison with the other Brucella species and strains. The inter- and intraspecies comparative analysis revealed several biovar-, haplotype- and strain-specific genetic polymorphisms that can implicate further genetic determinants related to host specificity and genomic specialization in B. suis. A set of chromosomal rearrangements and polymorphisms, including SNPs and INDELs, were found and will be further discussed in the following sections.

\section{Large chromosomal rearrangements in B. suis biovar 2 genomes}

The chromosomal organization of the six B. suis biovar 2 strains was examined by BamHI optical mapping and the pairwise alignment between optical and in silico maps allowed the identification of two major chromosomal rearrangements (one translocation event and one inversion) occurring in $B$. suis biovar 2 genomes, pointing out the degree of genome plasticity in B. suis species [11].

The $210 \mathrm{~kb}$ region translocated from Chr I to Chr II, associated to IS711, in the six biovar 2 studied genomes (Fig. 3) was firstly described in B. suis biovar 2 and 4 reference strains [32]. The translocated region shares $99 \%$ similarity with B.suis 1330 and encompasses genes with most functions assigned to processes such as transcription, replication and repair, carbohydrate metabolism and metabolism of co-factors and vitamins. Moreover, five tRNAs genes and six ribosomal proteins (L21, L27, L31, L32, L36 and S16) were moved to Chr II, and 14 coding regions in B. suis biovar 2 do not have orthologs in $B$. suis 1330 genome.

The most surprising rearrangement was the $944 \mathrm{~kb}$ chromosomal inversion present in strains PT09143, PT09172 and Bs143CITA (B. suis biovar 2 clade, subclade A1, Iberian clonal lineage) and covering $49 \%$ of Chr I [11]. Chr I encodes the majority of the core metabolic machinery for processes such as transcription, translation, and protein synthesis [42]. Although changing gene location, the majority $(>95 \%)$ of annotated coding regions were found to share $98-100 \%$ sequence identity with ATCC 23445 and both genomes from the Central-European clonal lineage.

At left and right crossover points, the inversion disrupted a Tripartite ATP-independent periplasmic (TRAP) dicarboxylate transporter, DctM subunit (C-terminus

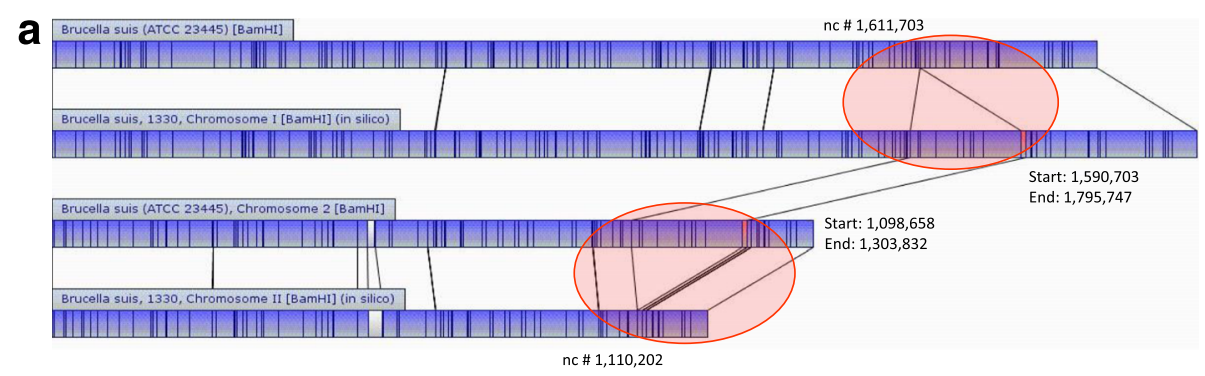

b

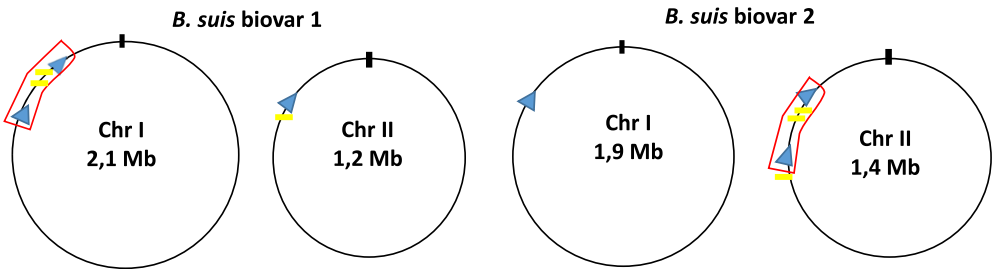

Fig. 3 Translocation event in B. suis biovar 2 strains. a Pairwise alignment of chromosome I and II optical and in silico maps for B. suis biovar 2 strain ATCC 23445 and B. suis biovar 1 strain 1330. Lines connecting two chromosomal maps indicate discontinuity in the alignment of fragments. The translocated region is highlighted in the red circles; unaligned restriction fragments, representing differences between two chromosomes, are showed in white; blue indicates aligned restriction fragments. $\mathbf{b}$ Schematic representation of both circular chromosomes. Open red box indicates the translocated region in chromosome I of $B$. suis biovar 1 and in chromosome II of $B$. suis biovar 2 ; blue triangles symbolize the $r$ rn loci; yellow boxes represents the insertion sequence 15711 
truncated, ortholog to ATCC 23445 BSUIS_A0375 and 1330 BR0344; nucleotide \#371383 in ATCC 23445), and an integral membrane protein TerC (N-terminus truncated, ortholog to ATCC 23445 BSUIS_A1382 and 1330 BR1332; nucleotide \#1316165 in ATCC 23445), respectively (Fig. 4). The TRAP transporters are a large family of substrate-binding protein (SBP)-dependent secondary transporters found in bacteria and archaea. These transporters have three domains that were defined on the basis of orthology to the three proteins that constitute the Dct system in Rhodobacter capsulatus [43], comprising an SBP of the DctP or TAXI families and two integral membrane proteins that form the DctQ and DctM protein families. Orthologs of the three genes can be found in all Brucella species (BSUIS_A0374 to BSUIS_A0376 orthologs in ATCC 23445) although $d c t Q$ and $d c t P$ were found to be variable within B. abortus, B. canis, and B. melitensis. The gene $\operatorname{dct} M$ is well conserved in Brucella spp. except in $B$. suis biovar 2 strains from Iberian ecovar, probably resulting in the inactivation of this gene in those strains. In B. melitensis $16 \mathrm{M}$ the Dct operon (BMEI1579BMEI11581) was predicted to be involved in the transport of mannitol although no experimental evidences exist, but in $R$. capsulatus, $d c t P$, dct $Q$, and $d c t M$ genes were shown to be essential for C4-dicarboxylate transport [43]. Mannose is both an important precursor in the $\mathrm{O}$-antigen biosynthetic pathway and in the production of the inner core moiety of lipopolysaccharide (LPS) [44, 45]. Loss of the ability to uptake mannitol can influence LPS structure and subsequently host immune responses. At the right crossover, the interrupted ORF codes for TerC, a protein possibly involved in tellurium resistance (inorganic ion transport and metabolism). This membrane protein harbors a CBS domain that is usually associated to enzymatic domains, membrane transporters or DNA-binding domains, playing an important role in host interactions.

\section{Distinctive SNPs of $B$. suis biovar 2 lberian ecovar and functional assessment}

The number of SNPs, including intergenic, missense and silent, obtained amongst the brucellae genomes relatively to B. suis ATCC 23445 are shown in Table 2 and in Additional file 6: Table S5. Eighty-two percent of the SNPs were identified in coding regions and an average of $51 \%$ correspond to missense mutations, affecting genes associated with different classes of cellular functions. The distribution of SNPs along the genome (SNPs per $200 \mathrm{~kb}$ ) showed no evident differences for the aforementioned chromosomal inversion at Chr I from Iberian B. suis biovar 2 strains (Additional file 7: Figure S2). The five B. suis biovar 2 strains shared 4087 SNPs. Two-hundred SNPs were unique to strains belonging to the Iberian ecovar (PT09143, PT09172 and Bs143CITA) and 228 discriminate the Central-European strains Bs364CITA and Bs396CITA. Among these, 102 missense SNPs were found exclusively in strains from Iberian ecovar. The three strains

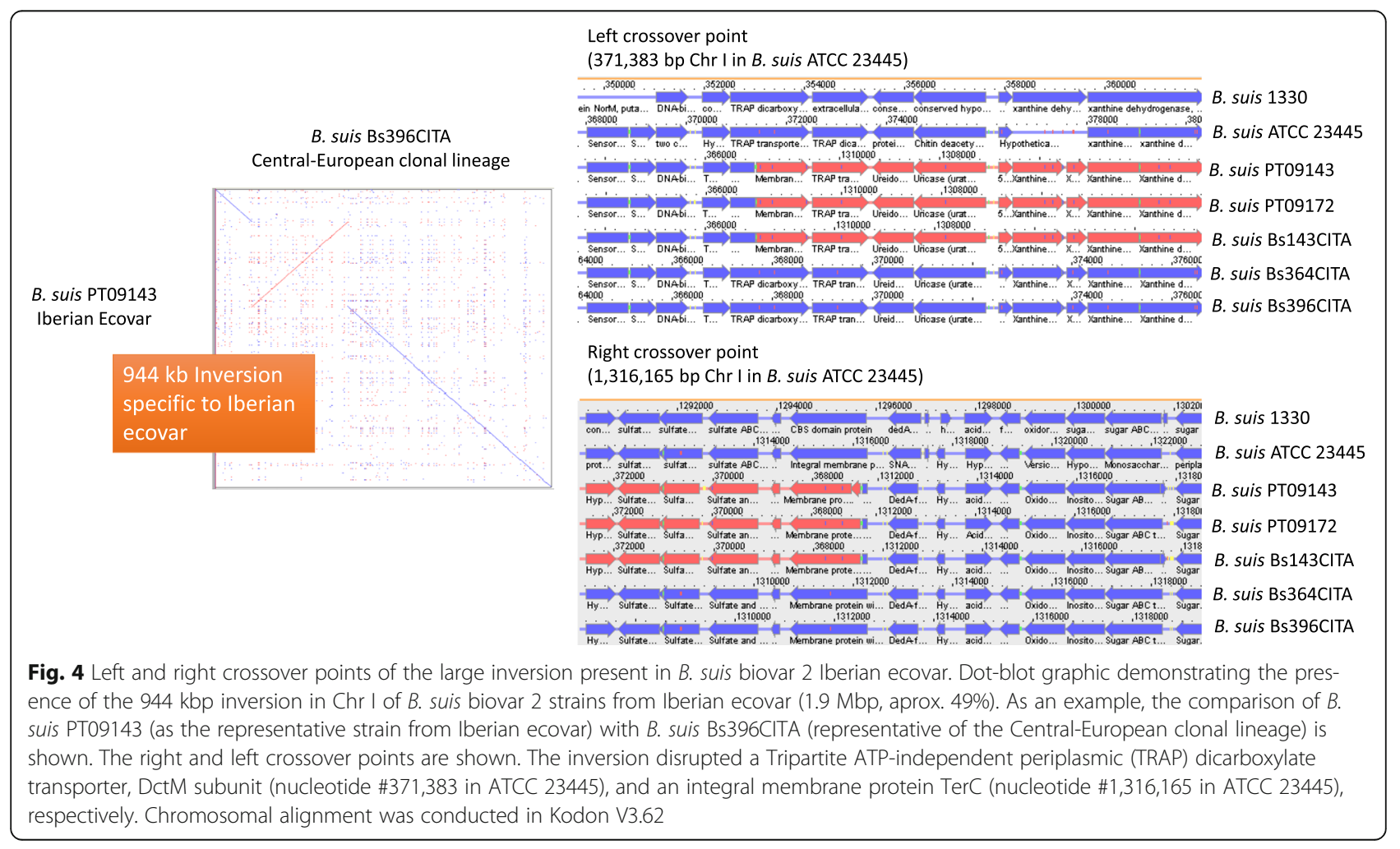


Table 2 Resume of mutation analysis of brucellae genomes using B.suis ATCC 23445 as reference

\begin{tabular}{|c|c|c|c|c|c|c|c|}
\hline \multirow[t]{2}{*}{ Strain Identification } & \multirow[t]{2}{*}{ Biovar } & \multirow[t]{2}{*}{ Host } & \multirow[t]{2}{*}{ INDELS } & \multicolumn{4}{|c|}{ Single Nucleotide Polymorphisms } \\
\hline & & & & Intergenic & Missense & Silent & Total \\
\hline B. suis ATCC 23445 & 2 & Hare & - & - & - & - & - \\
\hline B. suis $\mathrm{Bs} 364 \mathrm{CITA}$ & 2 & Wild boar & 11 & 165 & 256 & 146 & 567 \\
\hline B. suis Bs396CITA & 2 & Wild boar & 13 & 255 & 287 & 161 & 703 \\
\hline B. suis PT09172 & 2 & Wild boar & 18 & 225 & 420 & 260 & 905 \\
\hline B. suis PT09143 & 2 & Wild boar & 19 & 281 & 395 & 239 & 915 \\
\hline B. suis Bs143CITA & 2 & Wild boar & 20 & 295 & 434 & 268 & 997 \\
\hline B. suis VBI22 & 1 & Swine & 77 & 714 & 2687 & 1523 & 4924 \\
\hline B. suis 1330 & 1 & Swine & 78 & 729 & 2639 & 1557 & 4925 \\
\hline B. pinnipedialis B2/94 & not Applied & Dolphin & 100 & 763 & 2911 & 1517 & 5191 \\
\hline B. suis 686 & 3 & Seal & 77 & 764 & 2951 & 1661 & 5376 \\
\hline B. canis HSKA52141 & not applied & Dog & 72 & 799 & 2985 & 1632 & 5416 \\
\hline B. microti CCM 4915 & not applied & Dog & 80 & 985 & 2817 & 1644 & 5446 \\
\hline B. suis 513UK & not applied & Wild rodent & 75 & 840 & 3025 & 1596 & 5461 \\
\hline B. canis ATCC 23365 & 5 & Wild rodent & 71 & 826 & 2956 & 1706 & 5488 \\
\hline B. ceti TE10759-12 & not applied & Seal & 171 & 1002 & 3740 & 2017 & 6759 \\
\hline B. abortus 2308 & 1 & Cattle & 119 & 1152 & 4452 & 2465 & 8069 \\
\hline B. abortus 9-941 & 1 & Cattle & 121 & 1151 & 4465 & 2454 & 8070 \\
\hline B. abortus S19 & 1 & Vaccine & 118 & 1136 & 4478 & 2465 & 8079 \\
\hline B. melitensis ATCC 23457 & 2 & Goat & 104 & 1196 & 4662 & 2563 & 8421 \\
\hline B. melitensis M28 & not applied & Sheep & 104 & 1199 & 4687 & 2567 & 8453 \\
\hline B. ovis ATCC 25840 & 1 & Sheep & 225 & 1227 & 4529 & 2714 & 8470 \\
\hline B. melitensis M5-90 & 1 & Sheep & 108 & 1204 & 4707 & 2601 & 8512 \\
\hline B. melitensis $16 \mathrm{M}$ & 1 & Goat & 115 & 1117 & 5594 & 1994 & 8705 \\
\hline Brucella sp. 09RB8910 & not applied & African bullfrog & 207 & 6275 & 13,528 & 37,119 & 56,922 \\
\hline Brucella sp. 09RB8471 & not applied & African bullfrog & 260 & 6525 & 14,554 & 37,589 & 58,668 \\
\hline
\end{tabular}

of the Iberian ecovar showed a preferential functional enrichment of missense SNPs among the annotated genes associated to transporter/ efflux systems, other membrane receptors, metabolism processes, transcriptional regulators, regulatory proteins, cell replication, SOS response/DNA repair and ribosomal proteins (Fig. 5). The majority of discriminative mutations was associated to membrane related molecules $(29 \% ; n=30)$ and enzymes involved in catabolism process $(20 \% ; n=21)$, which may be related to molecular tropism to a specific environment or animal host.

\section{INDELs differentiative of biovar 2 lberian ecovar}

The comparative analysis of the effective INDELS disclosed 10 INDELs in coding regions, including one Large INDEL (LI $\geq 1 \mathrm{~kb}$ ), and three intergenic INDELs were found to be specific of the Iberian ecovar strains PT09143, PT09172 and Bs143CITA (Additional file 6: Table S7). The LI, with 1996 bp in Chr II (LI3234619), is presented in one region encoding ORFs BSUIS_B1354 to BSUIS_B1357 in ATCC 23445 within one Brucella flagellar gene cluster. BSUIS_B1354, BSUIS_B1355 and BSUIS_B1357 (BRA1127, BRA1128 and BRA1130 orthologs in 1330) code for hypothetical proteins without any specific functional domains. BSUIS_B1356 (BRA1129 ortholog in 1330) is predicted to code a flagellar protein FlgJ, possessing an $\mathrm{N}$-terminal domain responsible for proper rod assembly [46]. Primers (LI3234619-F and LI3234619-R) directed to flanking regions were used for INDEL search, confirming the presence of this INDEL in strains PT09143, PT09172, Bs143CITA and in the 104 biovar 2 strains from the Iberian ecovar. Although brucellae were considered non-motile bacteria for a long time, it has been reported that B. melitensis produces a functional flagellum with the characteristics of a sheathed flagellum-like structure, which is produced only transiently at the end of the exponential phase of growth [47, 48]. Moreover, recently "atypical" Brucella sp. strains were isolated from amphibians showing for the first time high motility $[18,49]$. The bacterial flagellum is a complex apparatus composed of at least 31 


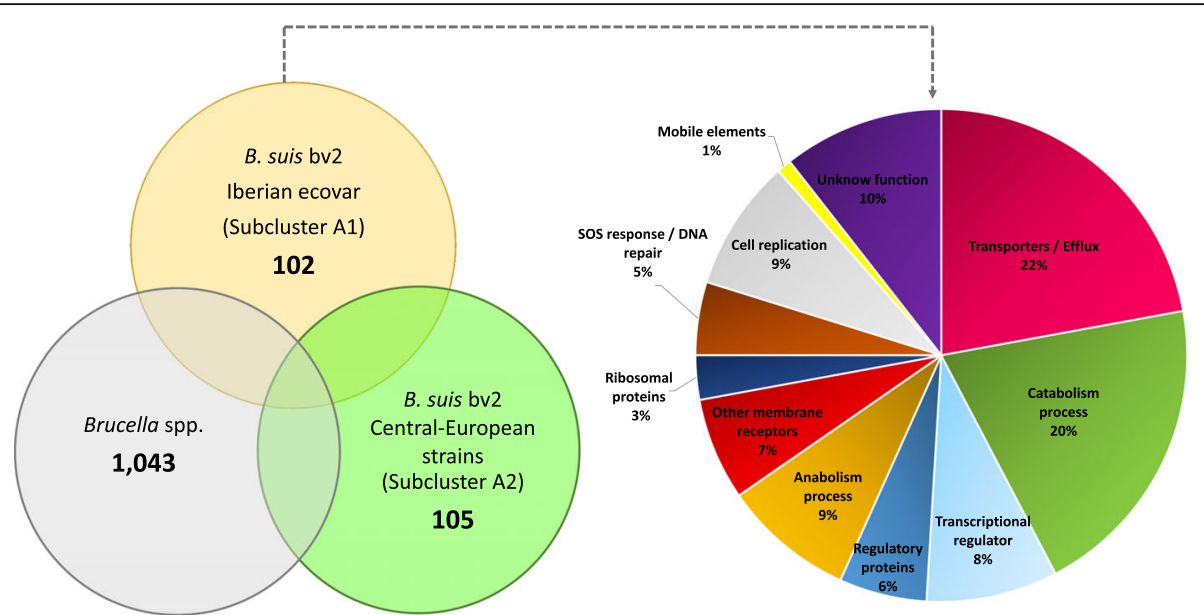

Fig. 5 Distribution of putative missense mutations between the two distinct $B$. suis biovar 2 ecovars. The Venn diagram shows the number of unique SNPs between B. suis biovar 2 Iberian ecovar (PT09143, PT09172 and Bs143CITA), B. suis biovar 2 Central-European lineage (Bs364CITA and Bs396(ITA) and the remaining Brucella spp. genomes. The associated pie chart shows the breakdown of the functional categories assigned to unique SNPs of Iberian ecovar

different proteins, which are similarly organized in all brucellae genomes, with genes distributed in three clusters on the small chromosome [18, 35]. However, a recent comparative gene-based analysis revealed that the majority of these flagellar genes were fully functional in all motile Brucella sp. strains analyzed, but non-motile brucellae presented a number of pseudogenized genes [18]. The $\mathrm{flg} J$ gene seems to be functional in all brucellae except in biovar 2 strains from the Iberian ecovar. It is documented that flagellar genes are required for the establishment of in vivo infection in mice and goats [42, 44]. Consequently, the inactivation (due to point mutations or small indels) or loss of key flagellar genes would influence the formation of a functional flagellum and therefore several functions can be affected, such as protein export or adhesion [50].

Among the nine Small INDELs (SI $<1-\mathrm{kb})$ in coding regions, three specific SIs were further evaluated due to their location in genome and eventual importance in evolution and genomic specialization of B. suis biovar 2 Iberian ecovar. SI79420 (678 bp) occurs within an ORF coding for an outer membrane protein ortholog to ATCC 23445 BSUIS_A0075 and 1330 BR0072, which was described as a putative autotransporter adhesin [35, 42]. In fact, different proteins belonging to the autotransporter family have been identified in Brucella genomes, sharing a common domain organization: an N-terminal secretion signal, a divergent and functional domain (passenger domain) and a conserved $\mathrm{C}$-terminal region $[35,51-53]$. The alignment of the ortholog genes in the different species indicated that this INDEL occurs within the passenger domain, showing a range in similarity (at the nucleotide level) with BSUIS_A0075, between 98\% (Bs364CITA and Bs396CITA), and 72\% (PT09143,
PT09172 and Bs143CITA). SI79420 probably caused the inactivation of the protein but it remains to be seen if this protein is functional or if differences within the passenger domain contribute to host or tissue specificity or clinical manifestations in wild boars or pigs. SI1627421 (59 bp) affects a permease, ortholog to ATCC 23445 BSUIS_A1714 and 1330 BR1873, associated to autotransporter proteins. The three strains from the Iberian clonal lineage presented the 59 bp INDEL next to the Cterminus. Since almost all of the known autotransporters are involved in functions related with the invasion process, the difference in the number of active autotransporters, and the variation within them, may play a role in the ability of each species to interact with its host and may thus be an important contributor to virulence [35, 42]. Lastly, SI2603410 (844 bp) represent an event that causes the elimination of one insertion sequence. The IS711 insertion sequence is unique to Brucella species and the number of copies in the genome varies between species and biovars. This is regarded as a key determinant in genome plasticity and was suggested to provide significant adaptive changes to genomes. Seven complete copies of this insertion sequence are recognized in $B$. suis 1330 and 13 in ATCC 23445 [34], as well as in strains Bs364CITA and Bs396CITA. However, Iberian ecovar strains PT09143, PT09172 and Bs143CITA present 12 copies of this insertion element, including orfA and $\operatorname{orf} B$ genes. From the three intergenic INDELs, the SI1356057 (88 bp) is located between a GntR family transcriptional regulator and a ketol-acid reductoisomerase. In ATCC 23445, a CDS coding for a hypothetical protein (BSUIS_A1430) is annotated among the GntR family transcriptional regulator (BSUIS_A1429), and the ketolacid reductoisomerase (BSUIS_A1431). Orthologous of 
the three genes are also present in both Central-European strains, but BSUIS_A1430 ortholog is missing in PT09143, PT0172 and Bs143CITA. Additionally, two intergenic SIs specific for strains PT09143 and Bs143CITA (Iberian ecovar, haplotype 2e), one in Chr I with $77 \mathrm{bp}$ (SI1423448), and other in Chr II with 47 bp (SI2041144) were found. Nevertheless, no frameshift is expected or no promoter region seemed to be affected by those two described INDELs.

The six abovementioned SIs were searched by targeted-PCRs in the 190 Brucella strains and it was confirmed that those events were specific of Iberian ecovar (Additional file 1: Table S1).

Finally, no INDELs were found to affect genes known to participate in virulence, such as lipopolysaccharide biosynthesis, two-component regulatory system BvrR/BvrS, type IV secretion system VirB or erythritol catabolic pathway.

\section{Conclusion}

In this work, a full genome comparative analysis of five $B$. suis biovar 2 strains isolated from wild boars belonging to the main circulating clonal lineages in the Iberian Peninsula and publicly available Brucella spp. genomes was performed. B. suis biovar 2 strains from the Iberian clonal lineage could be differentiated from strains from the Central-European clonal lineage not only by the presence of one large inversion in Chr I but also by a number of specific SNPs, deletions and insertions. Additionally, the mutational enrichment of the Iberian lineage was to genes encoding membrane proteins with potential of interaction with external stimulus, and to genes with impact on the pathogen metabolism. However, further studies are needs to understand the metabolic consequences of these disarrangements.

In summary, whole-genome analyses support that the B. suis biovar 2 Iberian clonal lineage evolved from the Central-European lineage and suggest that genomic specialization of this pathogen in the Iberian Peninsula is independent of a specific genomic event(s), but instead driven by allopatric speciation, resulting in the establishment of an ecovar.

\section{Additional files}

Additional file 1: Table S1. Brucella suis population genetic diversity information. (XLSX $29 \mathrm{~kb}$ )

Additional file 2: Table S2. List of genomes used for phylogenetic and comparative genomic analysis.) (DOCX $17 \mathrm{~kb}$ )

Additional file 3: De novo sequencing and assembly of B. suis biovar 2 strains PT09143, PT09172, Bs143CITA, Bs364CITA and Bs396CITA. (DOCX 24 kb)

Additional file 4: Table S3. Summary statistics for assembly of five $B$. suis biovar 2 strains isolates from wild boars; Table S4. General features of $B$. suis biovar 2 genomes. (XLSX $13 \mathrm{~kb}$ )

Additional file 5: Figure S1. Comparative chromosome mapping of 25 Brucella spp. genomes. Genomic alignment of concatenated chromosomes I and II was performed by superstretch approach: DNA seed 10 matches in windows size of 25 bases, minimal stretch length 60 bases, minimal cut-off for stretch identity of $60 \%$ in screening windows of 30 bases was used. Each cell in the matrix displays the identity score, with a corresponding color scale. The left-to-right diagonal of the matrix contains those cells representing the comparison of sequences compared to themselves. The value in each cell represent the percentage of repetitive regions for that sequence. The scale goes from black, corresponding with $100 \%$ identity, over blue towards white (0\% identity). Clustering analysis using UPGMA. All positions containing gaps and missing data were eliminated. (PDF $43 \mathrm{~kb}$ )

Additional file 6: Table S5. List of SNPs disclosed in the comparative genomic analysis of 25 Brucella genomes using as reference the annotated sequence of strain B. suis ATCC 23445; Table S6. List of INDELs disclosed in the comparative genomic analysis of 25 Brucella genomes using as reference the annotated sequence of strain $B$. suis ATCC 23445; Table S7. List of INDELs differentiative of B. suis biovar 2 Iberian ecovar. (XLSX 37286 kb)

Additional file 7: Figure S2. Distribution of SNPs along the genome (SNPs per 0,2 Mb). (PDF $194 \mathrm{~kb}$ )

\section{Abbreviations}

BLAST: basic local alignment search tool; CDS: coding DNA sequence; Chr: chromosome; GO: gene ontology; INDEL: insertions/deletions; InterPro: integrative protein signature database; KEGG: Kyoto encyclopedia of genes and genomes; LPS: lipopolysaccharide; ML: maximum likelihood; MLST: multiple locus sequence typing; MLVA: multiple-locus variable number tandem repeat analysis; MSA: multiple sequence alignment; MST: minimum spanning tree; NCBI: National Center for Biotechnology Information; NJ: neighbour-joining; ORFs: opening reading frames; PCR: polymerase chain reaction; RFLP: restriction fragment length polymorphism; SNP: single nucleotide polymorphism; UPGMA: unweighted pair group method using arithmetic averages; WG: whole genome

\section{Acknowledgments}

R.D. acknowledges the ongoing support from Faculdade de Ciências da Universidade de Lisboa.

\section{Funding}

This work was financed by project PTDC/CVT/104050/2008 and ARIMNET2/ 0003/2015 from Fundação para a Ciência e a Tecnologia (FCT). Support from Biosystems and Integrative Sciences Institute (BioISI, FCT/UID/Multi/04046/ 2013) is acknowledged. The funding bodies had no role in the design of the study, the collection, analysis and interpretation of data or writing of the manuscript.

\section{Availability of data and materials}

Most of the data generated during this study are included in this article and its supplementary information files. The remaining datasets generated are available in the NCBI GenBank repository, under accession no. NZ_CP007691/ NZ_CP007692 (BioProject PRJNA241449), NZ_CP007693/NZ_CP007694 (BioProject PRJNA242123), NZ_CP007695/NZ_CP007696 (BioProject PRJNA242205), NZ_CP007697/NZ_CP007698 (BioProject PRJNA242287), and NZ_CP007720/NZ_CP007721 (BioProject PRJNA242297), for chromosomes I/ II of PT09143, PT09172, Bs143CITA, Bs364CITA and Bs396CITA, respectively. The datasets used and/or analysed during the current study are available from the corresponding author on reasonable request.

\section{Authors' contributions}

ACF and RD carried out the genomic comparative analysis and wrote the manuscript. ACF carried out the experimental work. MICS, RT and RD conceived and planned the study, and revised the manuscript. All authors read and approved the final manuscript.

Ethics approval and consent to participate

This study has not directly involved humans, animals or plants.

Consent for publication

Not applicable. 


\section{Competing interests}

The authors declare that they have no competing interests.

\section{Publisher's Note}

Springer Nature remains neutral with regard to jurisdictional claims in published maps and institutional affiliations.

\section{Received: 26 January 2017 Accepted: 4 September 2017} Published online: 12 September 2017

\section{References}

1. Wattam AR, Williams KP, Snyder EE, Almeida NF Jr, Shukla M, Dickerman AW, Crasta OR, Kenyon R, Lu J, Shallom JM, Yoo H, Ficht TA, Tsolis RM, Munk C, Tapia R, Han CS, Detter JC, Bruce D, Brettin TS, Sobral BW, Boyle SM, Setubal JC. Analysis of ten Brucella genomes reveals evidence for horizontal gene transfer despite a preferred intracellular lifestyle. J Bacteriol. 2009;191(11):3569-79. https://doi.org/10.1128/JB.01767-08.

2. Foster JT, Beckstrom-Sternberg SM, Pearson T, Beckstrom-Sternberg JS, Chain PS, Roberto FF, Hnath J, Brettin T, Keim P. Whole-genome-based phylogeny and divergence of the genus Brucella. J Bacteriol. 2009;191(8): 2864-70. https://doi.org/10.1128/JB01581-08.

3. Whatmore AM, Perrett LL, MacMillan AP. Characterization of the genetic diversity of Brucella by multilocus sequencing. BMC Microbiol. 2007;7:34.

4. Le Flèche P, Jacques I, Grayon M, Al Dahouk S, Bouchon P, Denoeud F, Nockler K, Neubauer H, Guilloteau LA, Vergnaud G. Evaluation and selection of tandem repeat loci for a Brucella MLVA typing assay. BMC Microbiol. 2006;6:9.

5. EFSA. Scientific Opinion of the Panel on Animal Health and Welfare (AHAW) on a request from the Commission on porcine brucellosis (Brucella suis). The EFSA Journal. 2009;1144:1-112.

6. Godfroid J, Garin-Bastuji B, Saegerman C, Blasco JM. Brucellosis in terrestrial wildlife. Rev Sci Tech. 2013;32(1):27-42.

7. Duvnjak S, Račić I, Špičić S, Zdelar-Tuk M, Reil I, Cvetnić Ž. Characterisation of Brucella suis isolates from Southeast Europe by multi-locus variablenumber tandem repeat analysis. Vet Microbiol. 2015;180(1-2):146-50.

8. Kreizinger Z, Foster JT, Ronai Z, Sulyok KM, Wehmann E, Janosi S, Gyuranecz M. Genetic relatedness of Brucella suis biovar 2 isolates from hares, wild boars and domestic pigs. Vet Microbiol. 2014;172(3-4):492-8. https://doi. org/10.1016/jvetmic201405031.

9. Li ZJ, Cui BY, Chen H, Chen JD, Zhao HY, Piao DR, Jiang H, Zhang L, Tang X, Ke CW, Yao Z, Tian GZ. Molecular typing of Brucella suis collected from 1960s to 2010s in China by MLVA and PFGE. Biomed Environ Sci. 2013;26(6):504-8.

10. García-Yoldi D, Le Fleche P, De Miguel MJ, Muñoz PM, Blasco JM, Cvetnic Z, Marín CM, Vergnaud G, López-Goñi I. Comparison of multiple-locus variablenumber tandem-repeat analysis with other PCR-based methods for typing Brucella suis isolates. J Clin Microbiol. 2007;45(12):4070-2.

11. Ferreira AC, Dias R, Corrêa de Sá Ml, Tenreiro R. Whole-genome mapping reveals a large chromosomal inversion on Iberian Brucella suis biovar 2 strains. Vet Microbiol. 2016;192:220-5. doi:10.1016/j.vetmic.2016.07.024.

12. Muñoz PM, Boadella M, Arnal M, de Miguel MJ, Revilla M, Martínez D, Vicente J, Acevedo P, Oleaga A, Ruiz-Fons F, Marín CM, Prieto JM, de la Fuente J, Barral M, Barberán M, de Luco DF, Blasco JM, Gortázar C. Spatial distribution and risk factors of Brucellosis in Iberian wild ungulates. BMC Infect Dis. 2010;10(46) https://doi.org/10.1186/1471-2334-10-46.

13. Ferrão-Beck L, Cardoso R, Muñoz PM, de Miguel MJ, Albert D, Ferreira AC, Marín CM, Thiébaud M, Jacques I, Grayon M, Zygmunt MS, Garin-Bastuji B, Blasco JM, Sá MI. Development of a multiplex PCR assay for polymorphism analysis of Brucella suis biovars causing brucellosis in swine. Vet Microbiol. 2006;115(1-3):269-77.

14. Bäumler A, Fang FC. Host specificity of bacterial pathogens. Cold Spring Harb Perspect Med. 2013;3:a010041. doi:10.1101/cshperspect.a010041.

15. Ferreira AC, Tenreiro R, Corrêa de Sá MI, Dias R. Complete Genome Sequences of Three Iberian Brucella suis Biovar 2 Strains Isolated from Wild Boars. Genome Announc. 2014;2(4) doi:10.1128/genomeA00618-14.

16. Ferreira AC, Tenreiro R, Corrêa de Sá MI, Dias R. Complete Genome Sequences of Two Central European Brucella suis bv. 2 Haplotype 2c Strains Isolated from Wild Boars. Genome Announc. 2014;2(4) doi:10.1128/ genomeA00686-14.

17. Scholz HC, Revilla-Fernández S, Al Dahouk S, Hammerl JA, Zygmunt MS, Cloeckaert a, Koylass M, AM W, Blom J, Vergnaud G, Witte a, Aistleitner K,
Brucella HE. vulpis sp. nov., isolated from mandibular lymph nodes of red foxes (Vulpes vulpes). Int J Syst Evol Microbiol. 2016;66(5):2090-8.

18. Al Dahouk $S$, Köhler $S$, Occhialini $A$, Jiménez de Bagüés MP, Hammerl JA, Eisenberg T, Vergnaud G, Cloeckaert A, Zygmunt MS, Whatmore AM, Melzer F, Drees KP, Foster JT, Wattam AR, Scholz HC. Brucella spp. of amphibians comprise genomically diverse motile strains competent for replication in macrophages and survival in mammalian hosts. Sci Rep. 2017; doi:10.1038/ srep44420.

19. Eisenberg T, Riße K, Schauerte N, Geiger C, Blom J, Scholz HC. Isolation of a novel 'atypical' Brucella strain from a bluespotted ribbontail ray (Taeniura lymma). Antonie Van Leeuwenhoek. 2016;110(2):221-34. doi:10.1007/s10482016-0792-4.

20. Eisenberg T, Hamann HP, Kaim U, Schlez K, Seeger H, Schauerte N, Melzer F, Tomaso H, Scholz HC, Koylass MS, Whatmore AM, Zschock M. Isolation of potentially novel Brucella spp. from frogs. Appl Environ Microbiol. 2012; 78(10):3753-5

21. Alton $\mathrm{GC}$, Jones $\mathrm{LM}$, Angus $\mathrm{RD}$, Verger JM. Techniques for the brucellosis laboratory. Institute national de la Recherche Agronomique.1988. Paris. France.

22. Cloeckaert A, Verger JM, Grayon M, Grépinet O. Restriction site polymorphism of the genes encoding the major 25 and $36 \mathrm{kDa}$ outermembrane proteins of Brucella. Microbiology. 1995;141:2111-21.

23. Vizcaíno N, Verger JM, Grayon M, Zygmunt MS, Cloeckaert A. DNA polymorphism at the omp-31 locus of Brucella spp.: evidence for a large deletion in Brucella abortus, and other species-specific markers. Microbiology. 1997;143:2913-21.

24. Ashburner M, Ball CA, Blake JA, Botstein D, Butler H, Cherry JM, Davis AP, Dolinski K, Dwight SS, Eppig JT, Harris MA, Hill DP, Issel-Tarver L, Kasarskis A, Lewis S, Matese JC, Richardson JE, Ringwald M, Rubin GM, Sherlock G. Gene ontology: tool for the unification of biology. Nat genet. 2000;25(1):25-9. Online at Nature Genetics.

25. Hunter S, Apweiler R, Attwood TK, Bairoch A, Bateman A, Binns D, Bork P, Das U, Daugherty L, Duquenne L, RD F, Gough J, Haft D, Hulo N, Kahn D, Kelly E, Laugraud A, Letunic I, Lonsdale D, Lopez R, Madera M, Maslen J, McAnulla C, McDowall J, Mistry J, Mitchell A, Mulder N, Natale D, Orengo C, Quinn AF, Selengut JD, Sigrist CJ, Thimma M, Thomas PD, Valentin F, Wilson $\mathrm{D}, \mathrm{Wu} \mathrm{CH}$, Yeats C. InterPro: the integrative protein signature database. Nucleic Acids Res. 2009;37:D211-5. doi:10.1093/nar/gkn785.

26. Kanehisa M, Goto S. KEGG: Kyoto encyclopedia of genes and genomes. Nucleic Acids Res. 2000;28(1):27-30.

27. Götz S, García-Gómez JM, Terol J, Williams TD, Nagaraj SH, Nueda MJ, Robles M, Talón M, Dopazo J, Conesa A. High-throughput functional annotation and data mining with the Blast $2 \mathrm{GO}$ suite. Nucleic Acids Res. 2008;36(10):3420-35.

28. Tamura K, Stecher G, Peterson D, Filipski A, Kumar S. MEGA6: Molecular evolutionary genetics analysis version 6.0. Mol. Biol. Evolution. 2013;30:2725-9.

29. Chun J, Rainey FA. Integrating genomics into the taxonomy and systematics of the bacteria and Archaea. Int J Syst Evol Microbiol. 2014;64:316-24. doi:10. 1099/ijs.0.054171-0.

30. Halling SM, Peterson-Burch BD, Bricker BJ, Zuerner RL, Qing Z, Li LL, Kapur V, Alt DP, Olsen SC. Completion of the genome sequence of Brucella abortus and comparison to the highly similar genomes of Brucella melitensis and Brucella suis. J Bacteriol. 2005;187(8):2715-26.

31. Michaux-Charachon S, Bourg G, Jumas-Bilak E, Guigue-Talet P, AllardetServent A, O'Callaghan D, Ramuz M. Genome structure and phylogeny in the genus Brucella. J Bacteriol. 1997;179(10):3244-9.

32. Jumas-Bilak E, Michaux-Charachon S, Bourg G, O'Callaghan D, Ramuz M. Differences in chromosome number and genome rearrangements in the genus Brucella. Mol Microbiol. 1998;27(1):99-106.

33. Sankarasubramanian J, Vishnu US, Gunasekaran P, Rajendhran J. A genomewide SNP-based phylogenetic analysis distinguishes different biovars of Brucella suis. Infect Genet Evol. 2016;41:213-7. doi:10.1016/j.meegid.2016.04.012.

34. Audic S, Lescot M, Claverie JM, Scholz HC. Brucella microti: the genome sequence of an emerging pathogen. BMC Genomics. 2009;10:352. doi:10.1186/1471-2164-10-352 2009.

35. Chain PS, Comerci DJ, Tolmasky ME, Larimer FW, Malfatti SA, Vergez LM, Aguero F, Land ML, Ugalde RA, Garcia E. Whole-genome analyses of speciation events in pathogenic Brucellae. Infect Immun. 2005;73(12):8353-61.

36. Redelings BD, Suchard MA. Incorporating indel information into phylogeny estimation for rapidly emerging pathogens. BMC Evol Biol. 2007;7:40. 
37. Luan P-T, Ryder OA, Davis H, Zhang Y-P, Yu L. Incorporating indels as phylogenetic characters: impact for interfamilial relationships within Arctoidea (Mammalia: Carnivora). Mol Phylogenet Evol. 2013;66:748-56.

38. Ashkenazy H, Cohen O, Pupko T, Huchon D. Indel reliability in Indel-based phylogenetic inference. Genome Biol Evol. 2014;6:3199-209.

39. Georgiades K, Raoult D. Defining pathogenic bacterial species in the genomic era. Front Microbiol. 2010;1:151.

40. Zhang Y-Z, Li Y, Xie B-B, Chen X-L, Yao Q-Q, Zhang X-Y, Kempher ML, Zhou J, Oren A, Qin Q-L. Nascent genomic evolution and allopatric speciation of Myroides profundi D25 in its transition from Land to Ocean. mBio. 2016;7(1):e01946-15.

41. Ficht T. Brucella taxonomy and evolution. Future Microbiol. 2010;5:859-66.

42. It P, Seshadri R, KE N, JA E, JF H, TD R, RJ D, Umayam L, Lm B, MJ B, SC D, Rt D, AS D, JF K, Madupu R, WC N, Ayodeji B, Kraul M, Shetty J, Malek J, SE VA, Riedmuller S, Tettelin H, SR G, White O, SL S, DL H, LE L, SM H, SM B, CM F. The Brucella suis genome reveals fundamental similarities between animal and plant pathogens and symbionts. Proc Natl Acad Sci U S A. 2002;99(20):13148-53.

43. Forward JA, Behrendt MC, Wyborn NR, Cross R, Kelly DJ. TRAP transporters: a new family of periplasmic solute transport systems encoded by the dctPQM genes of Rhodobacter capsulatus and by homologs in diverse gram-negative bacteria. J Bacteriol. 1997;179(17):5482-93.

44. Cardozo PG, Macedo GC, Azevedo V, Oliveira SC. Brucella spp noncanonical LPS: structure, biosynthesis, and interaction with host immune system. Microb Cell Factories. 2006;5:13. https://doi.org/10.1186/1475-2859-5-13.

45. Zygmunt MS, Vizcaíno N, Cloeckaert A, Grilló MJ. Moriyón I \& López-Goñi I. Mutants in the lipopolysaccharide of Brucella ovis are attenuated and protect against $B$ ovis infection in mice Vet Res. 2014;45:72. https://doi.org/ 10.1186/s13567-014-0072-0.

46. Herlihey AF, Moynihan PJ, Clarke AJ. The essential protein for bacterial flagella formation FlgJ functions as a $\beta$-N-Acetylglucosaminidase. J Biol Chem. 2014;289(45):31029-42.

47. Ferooz J, Letesson JJ. Morphological analysis of the sheathed flagellum of Brucella melitensis. BMC Res Notes. 2010;3:333. doi:10.1186/1756-0500-3-333.

48. Fretin D, Fauconnier A, Köhler S, Halling S, Léonard S, Nijskens C, Ferooz J, Lestrate P, Delrue RM, Danese I, Vandenhaute J, Tibor A, DeBolle X, Letesson $\mathrm{JJ}$. The sheathed flagellum of Brucella melitensis is involved in persistence in a murine model of infection. Cell Microbiol. 2005;7(5):687-98.

49. Soler-Lloréns PF, Quance CR, Lawhon SD, Stuber TP, Edwards JF, Ficht TA, Robbe-Austerman S, O'Callaghan D, Keriel A. A Brucella spp. isolate from a Pac-Man frog (Ceratophrys ornata) reveals characteristics departing from classical Brucellae. Front Cell Infect Microbiol. 2016;6:116.

50. Haiko J, Westerlund-Wikström B. The role of the bacterial flagellum in adhesion and virulence. Biology (Basel). 2013;2(4):1242-67. doi:10.3390/biology2041242.

51. Ruiz-Ranwez V, Posadas DM, Estein SM, Abdian PL, Martin FA, Zorreguieta A. The BtaF trimeric autotransporter of Brucella suis is involved in attachment to various surfaces, resistance to serum and virulence. PLoS One. 2013:8(11): e79770. doi:10.1371/journal.pone.0079770.

52. Ruiz-Ranwez V, Posadas DM, Van der Henst C, Estein SM, Arocena GM, Abdian PL, Martín FA, Sieira R, De Bolle X, Zorreguieta A. BtaE, an adhesin that belongs to the trimeric autotransporter family, is required for full virulence and defines a specific adhesive pole of Brucella suis. Infect Immun. 2013;81(3):996-1007. https://doi.org/10.1128/IAl.01241-12.

53. Posadas DM, Ruiz-Ranwez V, Bonomi HR, Martín FA, Zorreguieta A. BmaC, a novel autotransporter of Brucella suis, is involved in bacterial adhesion to host cells. Cell Microbiol. 2012;14(6):965-82. doi:10.1111/j.1462-5822.2012.01771.x.

\section{Submit your next manuscript to BioMed Central and we will help you at every step:}

- We accept pre-submission inquiries

- Our selector tool helps you to find the most relevant journal

- We provide round the clock customer support

- Convenient online submission

- Thorough peer review

- Inclusion in PubMed and all major indexing services

- Maximum visibility for your research

Submit your manuscript at www.biomedcentral.com/submit
() BioMed Central 